\title{
The First Necessity of Progress
}

The United States spends almost twice as much of its gross domestic product on health care as other industrialized nations around the world, and this amount continues to rise. In return, outcomes achieved are disappointing, exemplified by a life expectancy that is 1.8 years less than average. ${ }^{1}$ This cost burden is viewed as unsustainable and has resulted in a massive and controversial effort to increase value by shifting from the traditional fee-for-service model to one that improves access to care and pays for results. The debate continues.

Respiratory therapists (RTs) have enjoyed a degree of notoriety for the value they provide to patients at the bedside, encompassing everything from out-patient rehabilitation services and home care to management of mechanical ventilation and therapist-directed treatment protocols. $^{2-4}$ In this issue of Respiratory CARe, Mummadi et $\mathrm{al}^{5}$ describe an RT-managed arterial catheterization and maintenance program in a 10-bed, community hospital ICU. This program tasked RTs with determining patient indication for arterial pressure lines, insertion and maintenance of the arterial catheter, and deciding need for arterial blood gas analysis. The authors conclude that "the study findings add to the substantial experience in which RTs can assume expanded roles in the care of ICU patients and can contribute to enhanced quality and patient safety, which is a major focus in health care." 5

All 3 of the arguments used to support the intervention-(1) optimizing intensivist efficiency and mitigating burn-out, (2) improving patient safety and utilization of services (arterial catheterization and blood gases), and (3) elevating respiratory care practice ("top of license" work)-support value (as defined by the equation, Value $=$ Benefit/Cost $)$.

Let us add perspective to each argument. First, there has been substantial discourse regarding a projected shortage of physicians in the years ahead. The Association of American Medical Colleges predicts that demand for physician

Dr Berlin has disclosed no conflicts of interest.

Correspondence: Thomas D Berlin DHSc RRT, Florida Hospital Orlando, 601 East Rollins Street, Orlando, FL 32803. E-mail: tom.berlin@flhosp.org.

DOI: $10.4187 /$ respcare. 05913 services will outstrip supply, leading to a projected shortfall of 40,800-104,900 physicians by the year $2030 .^{6}$ The aging of the baby boom generation and associated growth in the number of people living with chronic disease will

SeE the Original Study on Page 1520

largely drive this demand. ${ }^{7}$ Interestingly, the Health Resources and Services Administration projects the number of physicians and nurse practitioners trained to work in the ICU will be more than enough to meet the demand for critical care services through the year 2025, although regional variation will exist. ${ }^{8}$ This information, although seeming inconsistent, may reflect an increase in the percentage of new doctors selecting critical care residency training. Nevertheless, RTs must offer services wherever possible and practical to mitigate the burden of a provider shortfall.

In support of the second argument, it is well-documented that respiratory care centered upon evidence or expert opinion-based protocols has demonstrated better safety and efficacy. ${ }^{3,4,9,10}$ In the Mummadi et al study, ${ }^{5}$ the authors describe a success rate of $94.5 \%$ on initial arterial catheterization attempt by an RT and a reduction in arterial blood gas volume of $63.5 \%$ after introducing the RT-managed arterial line program. In a larger context, misallocation of services remains a problem, contributing to significant waste of precious health-care resources. It has been reported that up to $72 \%$ of respiratory care services are overordered, and up to $21 \%$ are underordered based upon established guidelines. ${ }^{11}$ A great opportunity awaits RTs who can expand their skill set and work diligently to optimize utilization of services based upon the benefit they provide to each individual patient. This will reduce waste and overall cost of care while enhancing the value of the $\mathrm{RT}$ at the bedside.

To the last point, Mummadi and team ${ }^{5}$ demonstrate that tasks traditionally performed by physicians within their hospital (arterial catheterization and line maintenance) can be done safely and effectively by RTs. This collaboration resulted in an expansion of RT scope of practice, adding to so-called top of license work. Although their hypothesis is simple, it establishes the basis for congruent thinking (ventilator liberation, endotracheal intubation, management of cardiac assist devices, extracorporeal membrane oxygenation administration, central line management, etc.) that 


\section{EDITORIALS}

Table 1. Typical Entry-Level Degree, Number of Jobs, Median Annual Wage, and Projected Job Growth of Nine NonPhysician Health Professions in the United States

\begin{tabular}{llrrc}
\hline \hline Profession & \multicolumn{1}{c}{$\begin{array}{c}\text { Entry } \\
\text { Degree }\end{array}$} & $\begin{array}{c}\text { Jobs } \\
(2014), \text { no. }\end{array}$ & $\begin{array}{c}\text { Wage } \\
(2016)\end{array}$ & $\begin{array}{c}\text { Growth } \\
(2014-2024), \%\end{array}$ \\
\hline Pharm & Doctorate & 297,100 & $\$ 122,230$ & 3 \\
PT & Doctorate & 210,900 & $\$ 85,400$ & 34 \\
PA & Master's & 94,400 & $\$ 101,480$ & 30 \\
OT & Master's & 114,600 & $\$ 81,900$ & 27 \\
SLP & Master's & 135,400 & $\$ 74,680$ & 21 \\
RN & Bachelor's & $2,751,000$ & $\$ 68,450$ & 16 \\
Diet & Bachelor's & 68,000 & $\$ 58,920$ & 14 \\
RT & Associate's & 120,700 & $\$ 58,670$ & 12 \\
Rad Tech & Associate's & 230,600 & $\$ 58,960$ & 9 \\
& & & &
\end{tabular}

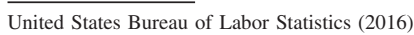

Pharm $=$ pharmacist

$\mathrm{PT}=$ physical therapist

$\mathrm{PA}=$ physician assistant

$\mathrm{OT}=$ occupational therapist

SLP $=$ speech language pathologist

$\mathrm{RN}=$ registered nurse

Diet $=$ dietician

$\mathrm{RT}=$ respiratory therapis

Rad Tech $=$ radiologic technologist

may lead to real optimization of the RT in the ICU. This work adds value to a health-care system overburdened with expense and inefficiency and represents opportunity for forward-thinking RTs.

In March of this year, Kacmarek and Walsh ${ }^{12}$ editorialized in this journal "We are clearly at a crossroads. Either we change radically and rapidly, or there is the real possibility that our profession will regress rather than progress. The status quo is not acceptable." According to the United States Bureau of Labor Statistics, the median annual wage for an RT in the United States is $\$ 58,670$, with job growth between 2014 and 2024 projected to be $12 \%$. Both numbers are at or near the bottom among 9 common, non-physician health professions (Table 1). ${ }^{13}$ It is worth noting that among these 9 professions, all but 2 (RT and radiology technician) typically require a baccalaureate degree or higher for entry into their profession (a bachelor's degree is now listed as the typical requirement for entry into nursing). To add to this concern, the National Center for Health Workforce Analysis has projected a 70\% increase in the supply of RTs between 2012 and 2025, significantly exceeding projected demand. ${ }^{14}$

RTs who wish to leave the field better than when they found it should not find the current state of the profession comforting. Thomas Edison is credited with the quote "Restlessness is discontent, and discontent is the first necessity of progress." 15

Without a clear and relentless focus on enhancing and communicating value to those we serve, our profession may regress in stature and, in this climate of cost reduction, risks irrelevance if not extinction. Some would argue the status quo is not only not acceptable; it may not long be sustainable.

Thomas D Berlin DHSc RRT Pulmonary and Respiratory Care Florida Hospital Orlando Orlando, Florida

\section{REFERENCES}

1. OECD Health Statistics 2017. Organization of Economic Cooperation and Development 2017. http://www.oecd.org/health/healthdata.htm. Accessed July 30, 2017.

2. Stoller JK. 2000 Donald F. Egan Scientific Lecture: are respiratory therapists effective? Assessing the evidence. Respir Care 2001;46(1): 56-66.

3. Kollef MH, Shapiro SD, Clinkscale D, Cracchiolo L, Clayton D, Wilner R, and Hossin L. The effect of respiratory therapist-initiated treatment protocols on patient outcomes and resource utilization. Chest 2000;117(2):467-475.

4. Stoller JK. The effectiveness of respiratory care protocols. Respir Care 2004;49(7):761-765.

5. Pack S, Hahn PY, Stoller JK, Mummadi SR. Respiratory therapymanaged arterial catheter insertion and maintenance program: experience in a non-teaching community hospital. Respir Care 2017; 62(12):1520-1524.

6. Association of American Medical Colleges. The complexities of physician supply and demand: projections from 2015 to 2030. IHS Markit 2017. https://aamc-black.global.ssl.fastly.net/production/ media/filer_public/a5/c3/a5c3d565-14ec-48fb-974b-99fafaeecb00/ aamc_projections_update_2017.pdf. Accessed August 6, 2017.

7. Anderson LA, Goodman RA, Holtzman D, Posner SF, and Northridge ME. Aging in the United States: opportunities and challenge for public health. Am J Public Health 2012;102(3):393-395.

8. National Center for Health Workforce Analysis, Health Resources and Services Administration. Health workforce projections: critical care physicians and nurse practitioners. US Department of Health and Human Services 2017. https://bhw.hrsa.gov/health-workforceanalysis/data. Accessed August 6, 2017.

9. Ely EW, Meade MO, Haponik EF, Kollef MH, Cook DJ, Guyatt GH, and Stoller JK. Mechanical ventilator weaning protocols driven by nonphysician health-care professionals. Chest 2001;120(6 Suppl):454S463S.

10. Blackwood B, Alderdice F, Burns K, Cardwell C, Lavery G, and O'Halloran P. Use of weaning protocols for reducing duration of mechanical ventilation in critically ill adult patients: Cochrane systematic review and meta-analysis. BMJ 2011;342:c7237. doi: 10.1136/bmj.c7237.

11. Stoller JK. Respiratory therapist-driven protocols: rationale and efficacy. West J Med 1997;167(6):408-410.

12. Kacmarek RM, and Walsh BK. The respiratory care profession is at a crossroads. Respir Care 2017;62(3):384-386.

13. Bureau of Labor Statistics. Occupational Handbook: healthcare occupations. US Department of Labor December 17, 2015. https:// www.bls.gov/ooh/. Accessed July 30, 2017.

14. National Center for Health Workforce Analysis, Health Resources and Services Administration. Health workforce projections: respiratory therapists. US Department of Health and Human Services 2016. https://bhw.hrsa.gov/health-workforce-analysis/data. Accessed August 6, 2017.

15. Edison TA. The diary and sundry observations of Thomas Alva Edison. Runes DD, editor. New York: Philosophical Library; 1948: 110. 